\title{
Quantum and classical complexity in coupled maps
}

\author{
Pablo D. Bergamasco* \\ Departamento de Física, CNEA, Libertador 8250, (C1429BNP) Buenos Aires, Argentina \\ and Departamento de Física, FCEyN, Universidad de Buenos Aires, C1428EGA, Argentina \\ Gabriel G. Carlo ${ }^{\dagger}$ and Alejandro M. F. Rivas ${ }^{\ddagger}$ \\ Departamento de Física, CNEA, CONICET, Libertador 8250, (C1429BNP) Buenos Aires, Argentina
}

(Received 18 October 2017; published 26 December 2017)

\begin{abstract}
We study a generic and paradigmatic two-degrees-of-freedom system consisting of two coupled perturbed cat maps with different types of dynamics. The Wigner separability entropy (WSE)—equivalent to the operator space entanglement entropy-and the classical separability entropy (CSE) are used as measures of complexity. For the case where both degrees of freedom are hyperbolic, the maps are classically ergodic and the WSE and the CSE behave similarly, growing to higher values than in the doubly elliptic case. However, when one map is elliptic and the other hyperbolic, the WSE reaches the same asymptotic value than that of the doubly hyperbolic case but at a much slower rate. The CSE only follows the WSE for a few map steps, revealing that classical dynamical features are not enough to explain complexity growth.
\end{abstract}

DOI: 10.1103/PhysRevE.96.062144

\section{INTRODUCTION}

Chaotic behavior is a classical property that implies the exponential divergence of close initial conditions. The ability to explore the whole available phase space, i.e., ergodicity, is indeed the main ingredient for statistical thermodynamics. On the other hand, quantum mechanics is governed by the Schrödinger equation, whose linearity forbids exponential divergences of close initial conditions. Also, entanglement is a quantum characteristic that has no classical counterpart. Consequently, as recently pointed out in Ref. [1], there is a battle between quantum and thermodynamic laws.

We mention a few contributions to this discussion. From the quantum to classical correspondence point of view, a pioneering work [2] has related the classically ergodic behavior with quantum entanglement production. Very recently a small quantum system of three superconducting qubits has been considered [3], showing a coincidence between regions of high (quantum) entanglement entropy and (classical) chaotic dynamics. On the other hand, entropy production in regular regions has been reported [4]. Also, in a Toda model of two interacting particles the chaotic and integrable cases could hardly be distinguished regarding entanglement generation [5]. Also, thermalization of quantum systems according to their type of dynamics is a subject of fundamental interest nowadays [6].

In order to perform an explicit comparison between quantum and classical mechanics it is of great help to have a quantity that can be calculated in both realms. Wigner functions represent quantum mechanics in phase space that provides a very suitable analog of Liouville distributions. Recently, in the spirit of algorithmic complexity, the Wigner separability entropy (WSE) [7] and the classical separability entropy (CSE) [8] have been introduced as measures of com-

\footnotetext{
*pablobergamasco@cnea.gov.ar

†carlo@tandar.cnea.gov.ar

†rivas@tandar.cnea.gov.ar
}

plexity of quantum and (discretized) classical distributions, respectively. In this work, we propose the notion of complexity to analyze correspondence by using these measures to study a two-degrees-of-freedom system. We consider two coupled perturbed cat maps, where one of them can be seen as the system and the other as the environment. The dynamics of these maps can be both hyperbolic (chaotic) (HH), both elliptic (regular) (EE), or mixed where one degree of freedom is hyperbolic and the other is elliptic (HE-EH).

We have found that for the $\mathrm{HH}$ case, the Wigner and Liouville distributions develop similar structures of increasing complexity, which are reflected in the WSE and the CSE hand-by-hand growth to a saturation value [9]. At the classical level, after an evolution of the order of the Ehrenfest time, the entropy decreases due to discretization. For the EE case, the quantum and classical measures do not always follow each other. The WSE and the CSE both reach lower values compared with the previous case. These results are similar to what it was found in Ref. [3] for chaotic and regular regions of phase space. Finally, for the mixed HE case the quantum complexity saturates at the same values of the $\mathrm{HH}$ case, although the growth rate is much slower. The classical complexity only grows during the first few map steps and then decreases, unable to reach the quantum asymptotics. In this way, we can observe that one hyperbolic degree of freedom is enough to generate high values of complexity (entanglement). The classical behavior differs and quantum mechanisms of complexity growth play a main role.

This paper is organized as follows: In Sec. II we explain the concepts of WSE and CSE and how they are used in our study. In Sec. III we present our model with a brief discussion on its properties. In Sec. IV we explain our results in detail and, in Sec. V we state our conclusions.

\section{WIGNER AND CLASSICAL SEPARABILITY ENTROPIES}

A state of a quantum system is described by means of the density operator $\hat{\rho}$ acting on the Hilbert space $\mathcal{H}$ such 
that $\operatorname{Tr}(\hat{\rho})=1$. This density operator is a vector belonging to the space $B(\mathcal{H})$ of Hilbert-Schmidt operators, where an inner product is defined as $\hat{A} \cdot \hat{B}=\operatorname{Tr}\left(\hat{A}^{\dagger} \hat{B}\right)$ such that the norm $\|\hat{\rho}\|=\sqrt{\operatorname{Tr}\left(\hat{\rho}^{2}\right)} \leqslant 1$. Decomposing the Hilbert space $\mathcal{H}$ as a tensor product $\mathcal{H}=\mathcal{H}_{1} \otimes \mathcal{H}_{2}$, the density operator has a Schmidt decomposition,

$$
\hat{\rho}=\sum \sigma_{n} \hat{a}_{n} \otimes \hat{b}_{n}
$$

with $n \in \mathbb{N}$, and where $\left\{\hat{a}_{n}\right\}$ and $\left\{\hat{b}_{n}\right\}$, such that $\operatorname{Tr}\left(\hat{a}_{m}^{\dagger} \hat{a}_{n}\right)=$ $\delta_{m n}, \operatorname{Tr}\left(\hat{b}_{m}^{\dagger} \hat{b}_{n}\right)=\delta_{m n}$, are orthonormal bases for $B\left(\mathcal{H}_{1}\right)$ and $B\left(\mathcal{H}_{2}\right)$, respectively. The Schmidt coefficients $\sigma_{1} \geqslant \sigma_{2} \geqslant$ $\ldots \geqslant 0$ satisfy $\sum_{n} \sigma_{n}^{2}=\operatorname{Tr}\left(\hat{\rho}^{2}\right)=\|\hat{\rho}\|^{2}$. The operator space entanglement entropy [10] is then defined as

$$
h[\hat{\rho}]=-\sum_{n} \tilde{\sigma}_{n}^{2} \ln \tilde{\sigma}_{n}^{2}, \quad \text { with } \tilde{\sigma}_{n} \equiv \frac{\sigma_{n}}{\|\hat{\rho}\|} .
$$

Among the several representations of quantum mechanics, the Weyl-Wigner representation performs a decomposition of the operators that act on the Hilbert space $\mathcal{H}$ on the basis spanned by $\widehat{R}_{x}$, the set of unitary reflection operators on points $\boldsymbol{x} \equiv(\boldsymbol{q}, \boldsymbol{p})[11,12]$ in a $2 d$-dimensional compact phase space $\Omega=\Omega^{\prime} \oplus \Omega^{\prime \prime}$. These reflection operators are orthogonal in the sense that

$$
\operatorname{Tr}\left[\hat{R}_{\boldsymbol{x}_{a}} \hat{R}_{\boldsymbol{x}_{b}}\right]=(2 \pi \hbar)^{d} \delta\left(\boldsymbol{x}_{b}-\boldsymbol{x}_{a}\right) .
$$

Hence, any operator $\hat{A}$ acting on the Hilbert space $\mathcal{H}$ can be univocally decomposed in terms of reflection operators as follows:

$$
\hat{A}=\left(\frac{1}{2 \pi \hbar}\right)^{d} \int d \boldsymbol{x} A_{W}(\boldsymbol{x}) \hat{R}_{\boldsymbol{x}} .
$$

With this decomposition, the operator $\hat{A}$ is mapped on a function $A_{W}(\boldsymbol{x})$, living in a $2 d$-dimensional compact phase space $\Omega$, the so called Weyl-Wigner symbol of the operator. Using Eq. (3) it is easy to show that $A_{W}(x)$ can be obtained by means of the trace operation

$$
A_{W}(\boldsymbol{x})=\operatorname{Tr}\left[\hat{R}_{\boldsymbol{x}} \hat{A}\right] .
$$

The Wigner function is defined in terms of the Weyl-Wigner symbol of the density operator,

$$
W(\boldsymbol{x})=(2 \pi \hbar)^{-d / 2} \rho(\boldsymbol{x})=(2 \pi \hbar)^{-d / 2} \operatorname{Tr}\left[\hat{R}_{x} \hat{\rho}\right] .
$$

Normalization of the density operator implies that

$$
\int d \boldsymbol{x} W(\boldsymbol{x})=\operatorname{Tr}(\hat{\rho})=1, \quad \text { while } \quad \int d \boldsymbol{x} W^{2}(\boldsymbol{x})=\|\hat{\rho}\| .
$$

Also, from the Schmidt decomposition of the density operator given in Eq. (1), we obtain the Schmidt (singular value) decomposition of the Wigner function:

$$
W(\boldsymbol{x})=\sum_{n} \sigma_{n} a_{n}\left(\boldsymbol{x}_{\mathbf{1}}\right) b_{n}\left(\boldsymbol{x}_{\mathbf{2}}\right),
$$

where $\left\{a_{n}\right\}$ and $\left\{b_{n}\right\}$ are now orthonormal bases for $L^{2}\left(\Omega_{1}\right)$ and $L^{2}\left(\Omega_{2}\right)$ (which are associated to the Hilbert space decomposition), such that

$$
a_{n}\left(\boldsymbol{x}_{\mathbf{1}}\right)=\operatorname{Tr}\left[\hat{R}_{\boldsymbol{x}_{1}} \hat{a}_{n}\right], \quad \text { and } \quad b_{n}\left(\boldsymbol{x}_{\mathbf{2}}\right)=\operatorname{Tr}\left[\hat{R}_{\boldsymbol{x}_{\mathbf{2}}} \hat{b}_{n}\right]
$$

The Wigner separability entropy is defined as [7]

$$
h[W]=-\sum_{n} \tilde{\sigma}_{n}^{2} \ln \tilde{\sigma}_{n}^{2},
$$

where

$$
\tilde{\sigma}_{n} \equiv \frac{\sigma_{n}}{\sqrt{\int d \boldsymbol{x} W^{2}(\boldsymbol{x})}} .
$$

The coefficients $\left\{\tilde{\sigma}_{n}\right\}$ in Eq. (6) are then the same than those in Eq. (2) and are the Schmidt coefficients of the singular value decomposition of $\tilde{W} \equiv W / \sqrt{\int d \boldsymbol{x} W^{2}(\boldsymbol{x})}$, such that $\tilde{W}$ is normalized in $L^{2}(\Omega): \int d \boldsymbol{x} \tilde{W}^{2}(\boldsymbol{x})=1$.

The WSE $h[W]$ quantifies the logarithm of the number of terms that effectively contribute to the decomposition of Eq. (5) and therefore provides a measure of separability of the Wigner function with respect to the chosen phase-space decomposition. Comparing Eq. (2) with Eq. (6), it is easy to see that the WSE is equal to operator space entanglement entropy [7], i.e., $h[W]=h[\hat{\rho}]$.

The main advantage of defining the separability entropy in phase space by means of the Wigner function is that such a quantity can be directly translated to classical mechanics. The classical analog of the Wigner separability entropy is the CSE (or s-entropy) $h\left[\rho_{c}\right]$ defined in Ref. [8], where a classical phase-space distribution $\rho_{c}(\boldsymbol{x})$ (discretized at the $\hbar$ scale) is used instead of the Wigner function $W(\boldsymbol{x})$. The CSE estimates the minimal amount of computational resources required to simulate the classical Liouvillian evolution and grows linearly in time for dynamics that cannot be efficiently simulated. Both the WSE and the CSE measure complexity of the distributions on the same footing. For our purposes this bridges the gap between quantum and classical mechanics.

It is worth mentioning that when the density operator $\hat{\rho}$ describes a pure state, $\hat{\rho}=|\psi\rangle\langle\psi|$, there exists a simple relation between the WSE and the entanglement content of the state $|\psi\rangle \in \mathcal{H}=\mathcal{H}_{1} \otimes \mathcal{H}_{2}$ [7]. In fact,

$$
h[W]=-2 S\left(\hat{\rho}_{1}\right)=-2 S\left(\hat{\rho}_{2}\right),
$$

where $\hat{\rho}_{1}=\operatorname{Tr}_{2}(\hat{\rho})$ and $\hat{\rho}_{2}=\operatorname{Tr}_{1}(\hat{\rho})$ are the reduced density operators for subsystems 1 and 2 , and $S$ is the von Neumann entropy. Since for a pure state $|\psi\rangle$ the von Neumann entropy of the reduced density matrix quantifies the entanglement $E$ of $|\psi\rangle[13,14]$,

$$
E(|\psi\rangle)=S\left(\hat{\rho}_{1}\right)=S\left(\hat{\rho}_{2}\right),
$$

the WSE is twice the entanglement entropy $E(|\psi\rangle)$ :

$$
h[W]=2 E(|\psi\rangle) .
$$

\section{MODEL SYSTEM}

Despite their simplicity, dynamical maps capture all the essential features of different types of complicated dynamical systems. This property and their relatively straightforward quantization turns them into a suitable tool to explore quantum to classical correspondence. The quantization of the cat map [15] (a paradigmatic linear automorphism on the torus and one of the most simple models of chaotic dynamics) has helped to elucidate many questions in the quantum chaos area [15-18]. We here investigate the behavior of two coupled perturbed cat 
maps, a two-degrees-of-freedom system. These two maps can have different types of dynamics.

Each degree of freedom is defined on the 2-torus as [15]

$$
\left(\begin{array}{c}
q_{t+1} \\
p_{t+1}
\end{array}\right)=\mathcal{M}\left(\begin{array}{c}
q_{t} \\
p_{t}+\epsilon\left(q_{t}\right)
\end{array}\right)
$$

with $q$ and $p$ taken modulo 1 , and

$$
\epsilon\left(q_{t}\right)=-\frac{K}{2 \pi} \sin \left(2 \pi q_{t}\right) .
$$

For the ergodic case we use the hyperbolic map

$$
\mathcal{M}_{h}=\left(\begin{array}{ll}
2 & 1 \\
3 & 2
\end{array}\right)
$$

while for regular behavior we choose the elliptic map

$$
\mathcal{M}_{e}=\left(\begin{array}{rr}
0 & 1 \\
-1 & 0
\end{array}\right)
$$

Quantum mechanics on the torus implies a finite Hilbert space of dimension $N=\frac{1}{2 \pi \hbar}$, where positions and momenta are defined to have discrete values in a lattice of separation $\frac{1}{N}$ [15]. In coordinate representation the corresponding propagator is given by a $N \times N$ unitary matrix

$$
U_{j k}=A \exp \left[\frac{i \pi}{N \mathcal{M}_{12}}\left(\mathcal{M}_{11} j^{2}-2 j k+\mathcal{M}_{22} k^{2}\right)+F\right],
$$

where

$$
\begin{gathered}
A=\left[1 /\left(i N \mathcal{M}_{12}\right)\right]^{1 / 2}, \\
F=[i K N /(2 \pi)] \cos (2 \pi j / N) .
\end{gathered}
$$

The states $\left\langle q \mid \mathbf{q}_{j}\right\rangle$ are periodic combs of Dirac $\delta$ distributions at positions $q=j / N \bmod (1)$, with $j$ integer in $[0, N-1]$.

The two-degrees-of-freedom system is defined in a fourdimensional phase space having coordinates $\left(q^{1}, q^{2}, p^{1}, p^{2}\right)$ [7] as

$$
\left(\begin{array}{c}
q_{t+1}^{1} \\
p_{t+1}^{1}
\end{array}\right)=\mathcal{M}_{1}\left[\begin{array}{c}
q_{t}^{1} \\
p_{t}^{1}+\epsilon\left(q_{t}^{1}\right)+\kappa\left(q_{t}^{1}, q_{t}^{2}\right)
\end{array}\right]
$$

and

$$
\left(\begin{array}{c}
q_{t+1}^{2} \\
p_{t+1}^{2}
\end{array}\right)=\mathcal{M}_{2}\left[\begin{array}{c}
q_{t}^{2} \\
p_{t}^{2}+\epsilon\left(q_{t}^{2}\right)+\kappa\left(q_{t}^{1}, q_{t}^{2}\right)
\end{array}\right]
$$

where the coupling between both maps is given by

$$
\kappa\left(q_{t}^{1}, q_{t}^{2}\right)=-\frac{K_{c}}{2 \pi} \sin \left(2 \pi q_{t}^{1}+2 \pi q_{t}^{2}\right) .
$$

The quantized version of the two-degrees-of-freedom system is obtained as the tensor product of the quantized one-degreeof-freedom maps, given by a $N^{2} \times N^{2}$ unitary matrix

$$
U_{j_{1} j_{2}, k_{1} k_{2}}^{2 D}=U_{j_{1} k_{1}} U_{j_{2} k_{2}} C_{j_{1} j_{2}}
$$

with the coupling matrix (diagonal in the coordinate representation)

$$
C_{j_{1} j_{2}}=\exp \left\{\left(\frac{i N K_{c}}{2 \pi}\right) \cos \left[\frac{2 \pi}{N}\left(j_{1}+j_{2}\right)\right]\right\}
$$

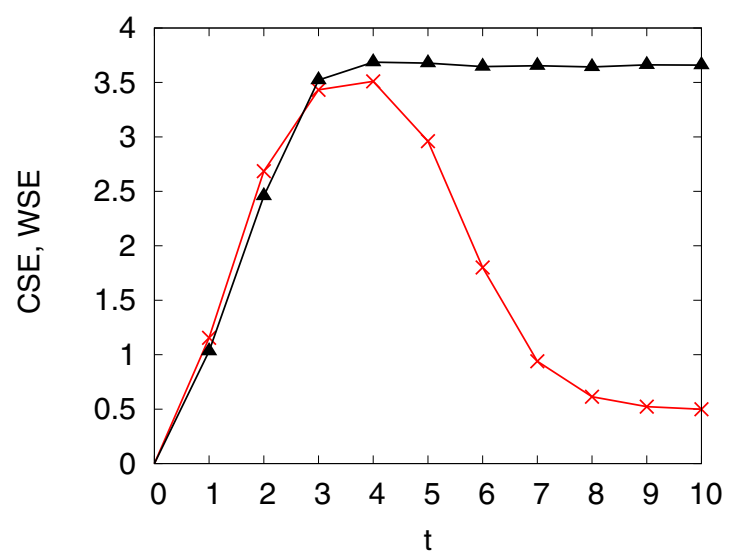

FIG. 1. CSE [(red) gray line with crosses] and WSE (black line with triangles) as a function of time $t$ (in map steps) in the HH case for $N=2^{6}$. Initial distributions are centered at $(q, p)=(0.5,0.5)$.

where $j_{1}, j_{2}, k_{1}, k_{2} \in\{0, \ldots, N-1\}$. We use $K=0.25$ and $K_{c}=0.5$ throughout our work, which guarantees the Anosov condition [16].

\section{RESULTS}

To investigate the quantum to classical correspondence regarding complexity growth, we study the evolution in time of $h[W]$ and of its classical counterpart $h\left[\rho_{c}\right]$. As initial states we have used a Gaussian phase-space distribution with dispersion equal to $\sqrt{\hbar}$ and its quantum analog, a coherent state on the torus, for both degrees of freedom. Profiting from the fact that the latter is a pure state we just compute the von Neumann entropy which is half the WSE. In the following, when we refer to WSE and CSE, we mean WSE/2 and CSE/2. We take $N=2^{6}$ for each map.

First, we consider the $\mathrm{HH}$ case with the initial distributions centered at $(q, p)=(0.5,0.5)$, which is a period 1 fixed point of both the hyperbolic and the elliptic maps. CSE and WSE as a function of time (map steps) are displayed in Fig. 1. The Liouville and Wigner distributions develop similar structures of increasing complexity as the evolution takes place. At time $t=3$, and after growing at a rate given by the average Lyapunov exponent, they show the maximum complexity where features of the stable manifold are still visible in the quantum case [see Figs. 2(a) and 2(b)]. From $t=3$ on the classical distribution becomes less complex due to discretization while the quantum one keeps its complexity through intertwined coherence patterns, as can be seen in Fig. 2(c) and 2(d). In case the classical distribution was not discretized, the CSE would continue to grow. The WSE grows until saturation at a value of the order of $\ln (0.6 \mathrm{~N})$, as predicted in Ref. [9]. At time $t=10$ the classical distribution is almost completely smoothed while the quantum one keeps the same morphology as the one at $t=4$ [see Figs. 2(e) and 2(f)]. We notice that we have removed the effects of the torus periodicity on the Wigner distributions in all figures [19].

The EE case is highly dependent on where the initial conditions are taken. We first show the evolution of the CSE and WSE as a function of time for distributions centered at the previous values, i.e. at the fixed point of period 1. As 

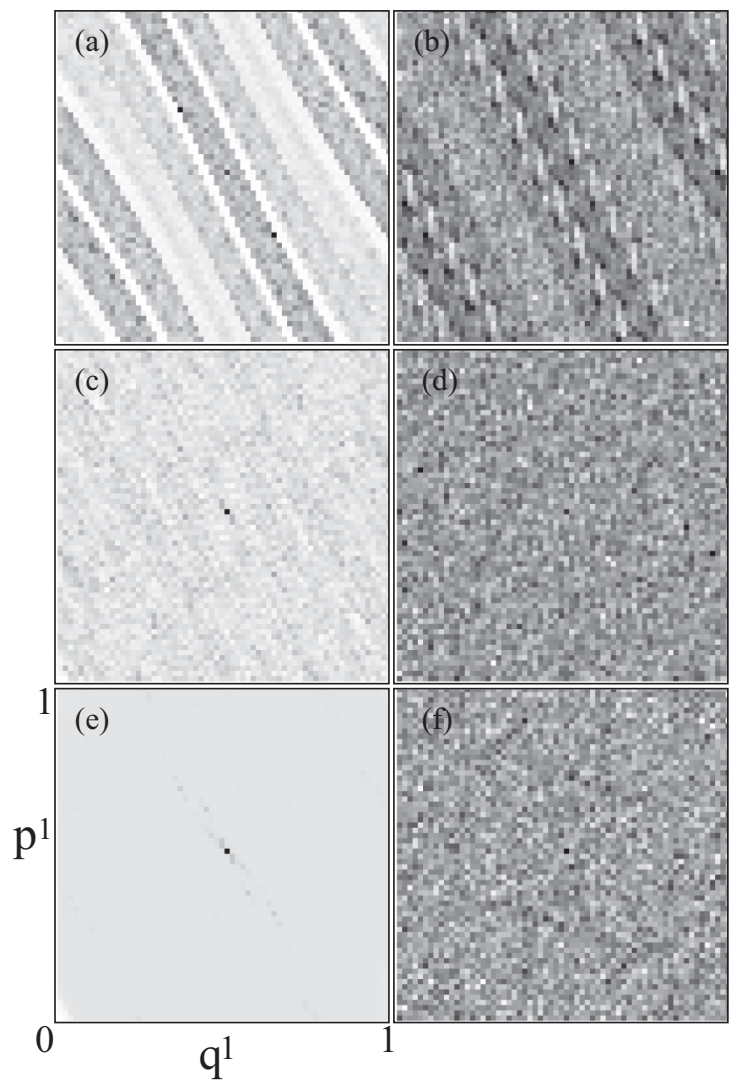

FIG. 2. Liouville and Wigner distributions at times $t=3[$ (a) and (b)], $t=4[$ (c) and (d)], and $t=10$ [(e) and (f)] for the HH case with initial conditions centered at $(q, p)=(0.5,0.5)$.

can be seen in Fig. 3, complexity does not grow significantly and the quantum and classical behavior is remarkably similar at all times (from $t=3$ on the agreement worsens). Small oscillations reflect the rotation of the distributions which do not explore much of the phase space. But if we select initial distributions centered at $(q, p)=(\pi / 4, \pi / 4)$, for instance (we just take a representative case from the maximum complexity ones), complexity grows as shown in Fig. 4. The saturation

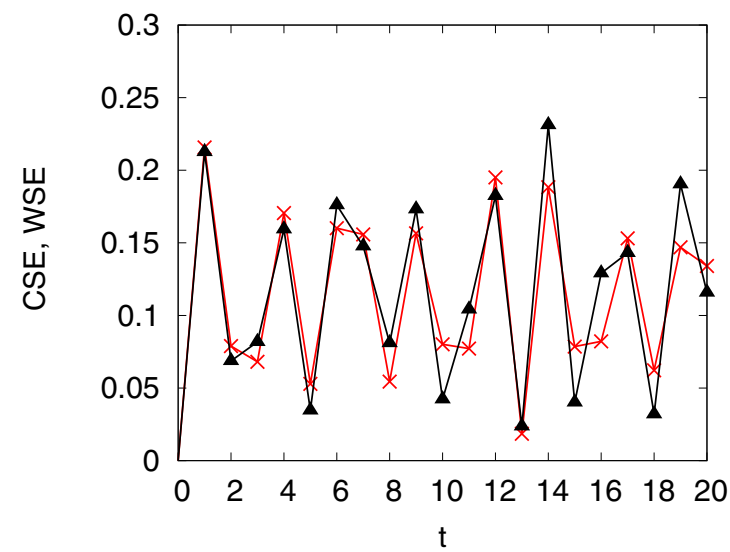

FIG. 3. CSE [(red) gray line with crosses] and WSE (black line with triangles) as a function of time $t$ (in map steps) in the EE case for $N=2^{6}$. Initial distributions are centered at $(q, p)=(0.5,0.5)$.

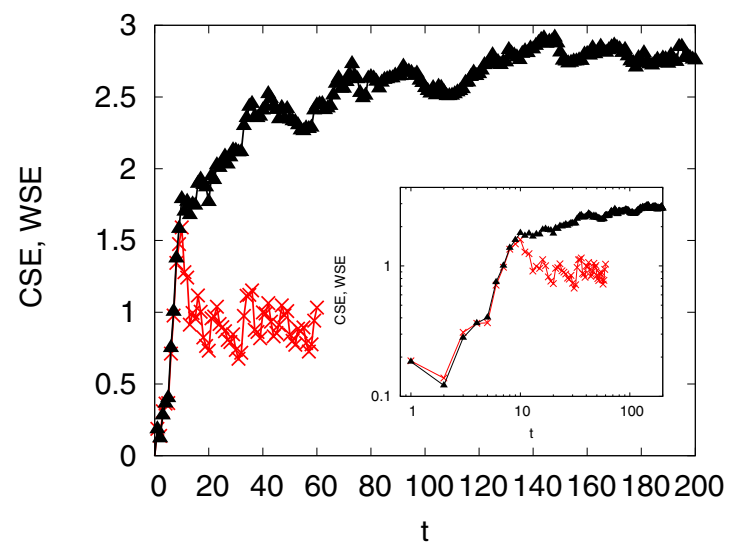

FIG. 4. CSE [(red) gray line with crosses] and WSE (black line with triangles) as a function of time $t$ (in map steps) in the EE case for $N=2^{6}$. Initial distributions are centered at $(q, p)=(\pi / 4, \pi / 4)$.

values of the WSE are always lower than the one for the $\mathrm{HH}$ case but greater than those of their corresponding CSE, suggesting that quantum effects begin to play an important role. In fact, from $t=10$ onward, the two curves take

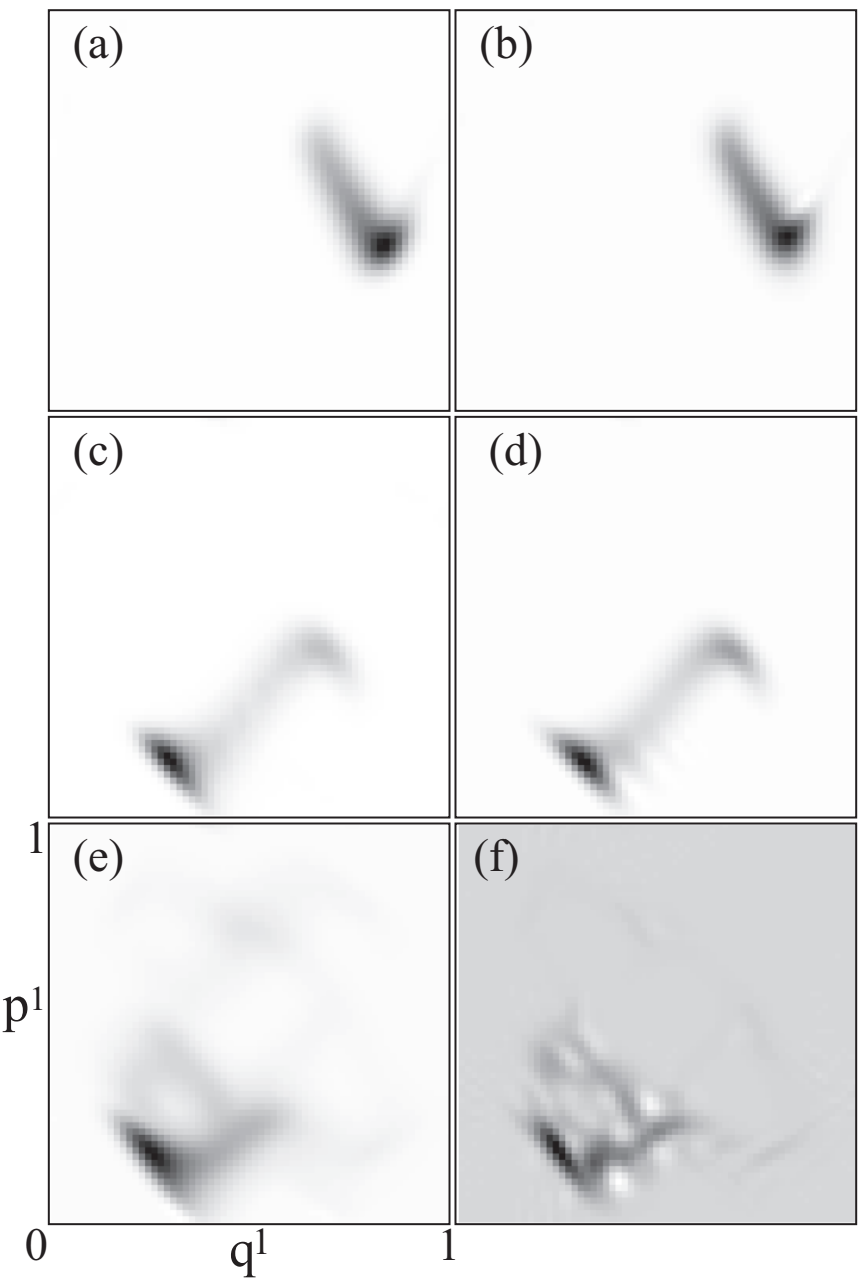

FIG. 5. Liouville and Wigner distributions at times $t=8[$ (a) and (b)], $t=11$ [(c) and (d)], and $t=50[(\mathrm{e})$ and (f)] for the EE case with initial conditions centered at $(q, p)=(\pi / 4, \pi / 4)$. 


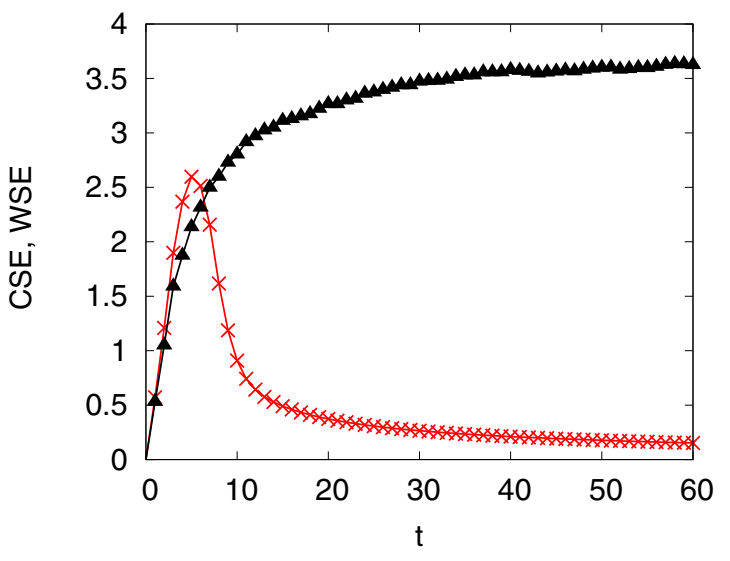

FIG. 6. CSE [(red) gray line with crosses] and WSE [black line with triangles] as a function of time $t$ (in map steps) in the HE case for $N=2^{6}$. Initial distributions are centered at $(q, p)=(0.5,0.5)$.

completely different behaviors. Moreover, the WSE reaches its maximum value after approximately 160 map steps, at a much slower rate than the $\mathrm{HH}$ case reflecting a different mechanism for complexity growth. In the inset we show the same quantities but in log-log scale. Here not only the power-law behavior becomes clear but also the abrupt change of slope at $t=10$. If we look at Fig. 5, then we can see how the quantum distribution develops interference fringes from $t=8$ [Fig. 5(b)] to $t=11$ [Fig. 5(d)]. The classical distribution just develops a secondary bulb of high density but of course coherences do not appear [see Figs. 5(a) and 5(c)]. Finally, for $t=50$ we can already see the typical morphology of the quantum distribution that has developed a lot of fringes in contrast with the classical one [see Figs. 5(e) and 5(f)]. It is interesting to note that until now everything seems to agree with the results of Ref. [3] regarding the entangling power of chaotic and regular regions of the classical phase space but keeping in mind this marked dependence on the initial conditions in the regular case.

Finally, we analyze the HE case for which we take the initial distributions centered at $(q, p)=(0.5,0.5)$. The WSE saturates at values similar to the HH case, although it takes a much longer time to reach them (see Fig. 6). The CSE only grows until $t=5$ and then decreases due to discretization. It is remarkable that just one hyperbolic degree of freedom is enough to reach maximum complexity, although at a classical level the dynamic is not completely ergodic. In this sense the behavior is strongly different than that of a mixed phase space with regular and chaotic regions. By looking at Fig. 7 it becomes clear that the Liouville distribution at times $t=3$ and $t=5$ [see Figs. 7(a) and 7(c)] develop more and more complex structures associated to the stable manifold, but that at $t=50$ [see Fig. 7(e)] it has already washed out almost all the details. The corresponding quantum distributions (for the same times) at the right panels show a different mechanism of complexity growth mainly based on coherences after $t=3$. It is worth noting that in order to perform all these comparisons the classical phase space has been truncated at the scale of $\hbar$; otherwise, the CSE would continue to grow as mentioned before. With this truncation our results show that the CSE starts to decay at times where the Wigner function begins to develop

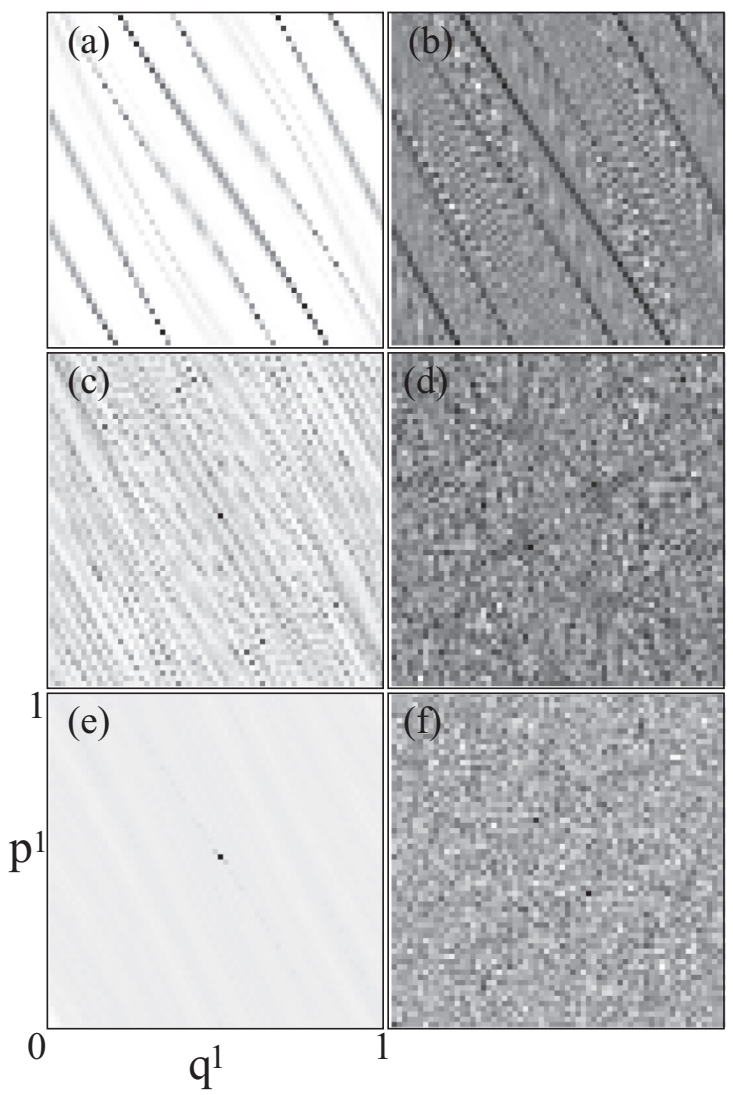

FIG. 7. Liouville and Wigner distributions at times $t=3[$ (a) and (b)], $t=5[$ (c) and (d)], and $t=50[(\mathrm{e})$ and (f)] for the HE case with initial conditions centered at $(q, p)=(0.5,0.5)$.

coherences, which have no classical meaning. For example, from Fig. 4 we observe that the CSE and the WSE start to separate at $t=10$, when coherent effects start to appear in the Wigner distributions shown in Fig. 5.

\section{CONCLUSIONS}

We have studied a generic system consisting of two coupled perturbed cat maps, considering the doubly hyperbolic, elliptic, and the mixed cases. By using the WSE and the CSE as two sides of the same complexity notion we find that for the $\mathrm{HH}$ case, the quantum and classical complexity growth share the same behavior (despite discretization effects of the classical distribution). The WSE and the CSE reach the maximum theoretical limit predicted in Ref. [9], which is at the order of $\ln (0.6 N)$. In the EE case this quantum to classical correspondence depends on the initial conditions, but the HH case complexity maximum is not reached. This confirms recent findings published in Ref. [3] regarding the production of entanglement in chaotic and regular regions of the phase space. But it is important to clarify that entanglement entropy generation and classical chaotic or regular behavior are directly related through the complexity notion in these cases. Thus the connection is not surprising.

Moreover, this is not always the case, as, for instance, in the nongeneric baker map, which is chaotic but not 
complex [7]. In the $\mathrm{HE}$ case of our generic system, the WSE reaches the maximum theoretical value around $\ln (0.6 N)$, similarly to the $\mathrm{HH}$ case, although the time of the transient is much longer. The CSE does not reach this value and has a markedly different behavior. This reveals that in the mixed scenario the quantum mechanisms of complexity growth (namely coherences) play a central role. On the other hand, just one hyperbolic degree of freedom is enough to reach maximum complexity, despite the fact that the dynamics is not completely ergodic at the classical level. It is interesting to mention the connection of this finding with the concept of "weak quantum ergodicity" [20]. This notion states that for "slow ergodic" systems (i.e., those in which the classical rate of spreading is sufficiently slow) the patterns of quantum transport can be well understood by superimposing diffractive spreading on top of the classically allowed motion.
Our results provide a wider picture of complexity growth, including mixed dynamics scenarios. These findings suggest new experiments with controllable quantum systems, where the behavior of each component could be selected to be regular or chaotic. In the future, we will study the role played by complex eigenvalues of the symplectic matrix leading to loxodromic behavior [21]. On the other hand, the perturbed cat maps used in this work are paradigmatic general models of dynamical systems. They have been extensively used in the literature as such. However, in order to prove the universality of our results, we plan to perform similar studies for other systems in the future.

\section{ACKNOWLEDGMENT}

Support from CONICET is gratefully acknowledged.
[1] D. Castelvecchi, Nature 543, 597 (2017).

[2] A. K. Pattanayak, Phys. Rev. Lett. 83, 4526 (1999).

[3] C. Neill et al., Nat. Phys. 12, 1037 (2016).

[4] M. Lombardi and A. Matzkin, Phys. Rev. E 83, 016207 (2011).

[5] G. Casati, I. Guarneri, and J. Reslen, Phys. Rev. E 85, 036208 (2012).

[6] F. Borgonovi et al., Phys. Rep. 626, 1 (2016).

[7] G. Benenti, G. G. Carlo, and T. Prosen, Phys. Rev. E 85, 051129 (2012).

[8] T. Prosen, Phys. Rev. E 83, 031124 (2011).

[9] J. N. Bandyopadhyay and A. Lakshminarayan, Phys. Rev. Lett. 89, 060402 (2002).

[10] P. Zanardi, Phys. Rev. A 63, 040304(R) (2001); X. Wang and P. Zanardi, ibid. 66, 044303 (2002); M. A. Nielsen, C. M. Dawson, J. L. Dodd, A. Gilchrist, D. Mortimer, T. J. Osborne, M. J. Bremner, A. W. Harrow, and A. Hines, ibid. 67, 052301 (2003); T. Prosen and I. Pižorn, ibid. 76, 032316 (2007).

[11] A. M. O. de Almeida, Phys. Rep. 295, 265 (1998).
[12] A. M. F. Rivas and A. M. O. de Almeida, Ann. Phys. 276, 223 (1999).

[13] M. A. Nielsen and I. L. Chuang, Quantum Computation and Quantum Information (Cambridge University Press, Cambridge, 2000).

[14] G. Benenti, G. Casati, and G. Strini, Principles of Quantum Computation and Information (World Scientific, Singapore, 2007), Vol. II.

[15] J. H. Hannay and M. V. Berry, Physica D 1, 267 (1980).

[16] M. B. De Matos and A. M. O. de Almeida, Ann. Phys. 237, 46 (1995).

[17] F. Haake, Quantum Signatures of Chaos (Springer-Verlag, New York, 2001).

[18] M. D. Espositi and B. Winn, J. Phys. A: Math. Gen. 38, 5895 (2005).

[19] A. Arguelles and T. Dittrich, Physica A 356, 72 (2005).

[20] L. Kaplan and E. J. Heller, Physica D 121, 1 (1998).

[21] A. M. F. Rivas, M. Saraceno, and A. M. O. de Almeida, Nonlinearity 13, 341 (2000). 